\title{
The Role of Individual Differences in Executive Attentional Networks and Switching Choices in Multi-Task Management
}

\author{
Robert S. Gutzwiller ${ }^{1}$, Christopher D. Wickens ${ }^{2,3}$, \& Benjamin A. Clegg ${ }^{2}$ \\ ${ }^{I}$ Space and Naval Warfare Systems Center Pacific \\ ${ }^{2}$ Colorado State University \\ ${ }^{3}$ Alion Science and Technology
}

\begin{abstract}
Individual differences in cognitive processing relate to critical performance differences in real-world environments. Task switching is required for many of them and especially for task management during overload. Research exploring individual differences related to switching behavior (both frequency, and adherence to "optimal" switch times) is, however, sparse. We examined these relationships here, using the attentional network task to index executive control, and an ongoing tracking task (within a larger suite of concurrent task demands) to examine switching behavior. The results failed to support a general relationship between executive control and frequency in a complex, heterogeneous multi-task environment. However, higher executive control participants more successfully exploited "optimal" switching times, highlighting the varying role of individual differences in task management, when choice is unconstrained.
\end{abstract}

The environment surrounding the astronaut is complex, dynamic, and requires a great deal of task management. In particular, astronauts are confronted with choices concerning where they direct attention, and which of many different tasks they perform at any given moment (but particularly under high cognitive load, our interest here). Under these circumstances, operators are routinely forced into tradeoff situations pitting allocating attention toward one task at the expense of removing attention from another. This choice occasionally results in accidents, even when operators possess large amounts of experience (Chou, Madhavan, \& Funk, 1996; Funk, 1991). Most notoriously, prospective memory failures the result of frequent interruptions, and thus frequent task switches (Dismukes \& Berman, 2010; Loukopoulos, Dismukes, \& Barshi, 2009) are linked to these accidents, suggesting that task switching plays a large role in complex operations.

While interruption and frequent switching are a potential causal factor in incidents, at the other end of the spectrum, the failure to switch, or cognitive tunneling (Dehais, Causse, \& Tremblay, 2011; Wickens et al., 2013) can also be harmful. Operators may inappropriately allocate attention to a particularly engaging task, to the exclusion of others (Wickens \& Alexander, 2009), a "time-on-task" effect. Because the time on task element has appeared as a critical part of the STOM switching model (Wickens, Gutzwiller, \& Santamaria, 2015), we have considered the different kinds of time on task effects that may occur, and describe relevant types below.

On the one hand, there are monotonic effects, lasting throughout the entire task, such as boredom or potentially resource depletion (Kurzban et al., 2013). Switch avoidance may be high especially under high workload, when resources are scarce (Gutzwiller et al. 2014), and this may be the case for long periods of time. On the other hand, we have also considered more periodic effects on switch likelihood. One prototype is the completion of the sub goal of a larger task (Altmann \& Trafton, 2002; Bogunovich \& Salvucci, 2008), which presents an optimal time for switching. Our current focus is on these more transient, "natural breakpoints" that occur throughout a given task. When such periods of opportunity for switching occur, they provide a natural decrease in difficulty, and the likelihood that operators switch away from their ongoing task should increase (e.g., Bogunovich \& Salvucci, 2008). Our approach is to apply this logic to a continuous tracking task, a novel application but one similar to steering control in a vehicle (e.g., Salvucci \& Taatgen, 2011).

Our dual interest in individual differences suggests individuals might differ in their ability to exploit such natural switching opportunities. However, predictions for such a relationship are remarkably challenging because of the complex relationship between switching behavior, resources, working memory (WM) and executive control (EC), as we further discuss below.

\section{Individual Differences in Executive Attention}

One a priori assumption concerning task switching is that it requires mental resources. In similar applied conditions, task switching appears to be reduced when the overall mental workload of the task ensemble is increased (e.g., Gutzwiller et al., 2014; Sebok et al., 2015). These results also agree with results from the more basic voluntary task switching literature, which considers simple cognitive tasks rather than complex task performance (e.g., Demanet, Verbruggen, Liefooghe, \& Vandierendonck, 2010; Weywadt \& Butler, 2013).

The resources demanded for such a switch might be reasonably assumed to be related to $\mathrm{WM}$, as it is taxed to concurrently keep place on any interrupted tasks for task resumption, but also choose opportune moments to switch. These resource and WM demands are especially strong within complex task performance. Together, we suggest some amount of WM, or executive control is required for a switch event. Increased capacity could be assumed to lead to either more switching overall (as resources are less constrained) or more frequent switching at more opportune times (increased exploitation). However the former has failed to accumulate much evidence in voluntary task switching experiments, at least related to WM capacity (Butler, Arrington, \& Weywadt, 2011). The latter has gone completely unexamined. 
It can also be argued quite plausibly that executive control is involved in switching, given the requirements for planning a switch. Arrington and Yates (2009) used the attentional network task (ANT; Fan et al., 2002) which is a combination of a cueing task and a more traditional Flanker task (Eriksen \& Eriksen, 1974) to assess attention in their voluntary task switching experiment. The executive control network component of the ANT is based on evidence of a brain network that is involved in resolving response conflicts, a component often tapped in other measures such as the Stroop task (e.g., McLeod, 1991). Results from Arrington and Yates (2009) suggested with worse conflict resolution in the Flanker task (indicating lower EC), there was a decreased likelihood to switch tasks. Despite this finding, it is not clear whether EC could also be related to switching at more opportune times (the exploiting hypothesis).

Combined, the exhibited roles of individual differences, resources, and switching are somewhat contradictory. In some cases, performance on conflict resolution items, presumably tapping some executive functioning, is related to decreased switching. But in other cases, reduced WM resources (induced by cognitive load) also reduced switches. These are contradictory results from an individual differences perspective, because increased WM capacity has been associated with improved executive control on tasks like Stroop (Kane \& Engle, 2003).

The relationship of switching with executive control is complicated by the definition of EC (Banich, 2009). While WM capacity is a facet of EC, so too is the resistance to distraction or the ability to focus attention. To the extent these are similar, the available predictions contradict: higher EC would be related to higher WM, and thus more resources should lead to more switching; or, paradoxically by increasing the resistance to distraction, higher EC could actually increase the resistance to switching (as an alternative task would be less likely to capture attention). While the above is unclear whether individual differences in EC may, or may not predict switching frequency, it does suggest that greater EC predicts more opportunistic switches through the ability to better control attention, in support of the exploitation hypothesis.

The present experiment builds on the past work by using unconstrained task switching instructions, and a complex multi-task experimental platform (Gutzwiller et al., 2014). Therefore, our task environment is more similar to that of an astronaut with multiple, complex tasks. Work in this area provides unique contributions to knowing on task switching, which has relied on basic, and often few (less than 3) tasks.

The two primary goals of this examination are, first, to address the role of individual differences in conflict resolution as they relate to switching frequency. Second, we examine whether or not individual differences would be predictive of exploiting optimal switching behaviors (e.g., switching during a natural breakpoint, or not).

Our first hypothesis concerned the relationship between individual differences in attentional networks (assessed by the ANT task) and switching behavior. We predicted $(\mathrm{HI})$ that participants who are worse at conflict resolution, which taps the executive control network, would switch less in general during the MATB trials. This decreased switching would lead to a negative correlation between the ANT measure and switching frequency, as was found in Arrington and Yates (2009). However, if prior results are due to adherence to constraints on decisions, imposed by voluntary task switching instructions (e.g., Arrington \& Logan, 2004), these relationships may not exist in our less constrained settings which required complex, realistic task performance.

Our second hypothesis was (H2) that operators with worse conflict resolution would be associated with lowered frequency of switching at optimal switching points (poorer exploitation), and a correspondingly reduced frequency of switching during non-optimal points. Thus, we expected conflict resolution efficiency could impact switching at optimal points, but not necessarily switching frequency overall. We examined this hypothesis by using state space modeling of a continuous tracking task.

\section{METHOD}

\section{Participants}

Seventy participants from Colorado State University participated for optional, partial course credit, or were paid an hourly rate of $\$ 10$ for participation over two hours. No monetary incentives were offered for performance.

\section{Materials}

The Multi-Attribute Task Battery II (MATB II; Santiago-Espada, Myer, Latorella, \& Comstock, 2011) platform was used. MATB II is based on MATB (Comstock \& Arnegard, 1992) and was provided by NASA. Participants made all responses on a standard Dell computer running Windows 7, using a Logitech joystick (for the tracking task) or a standard mouse. Audio information was presented over a set of Phillips headphones.

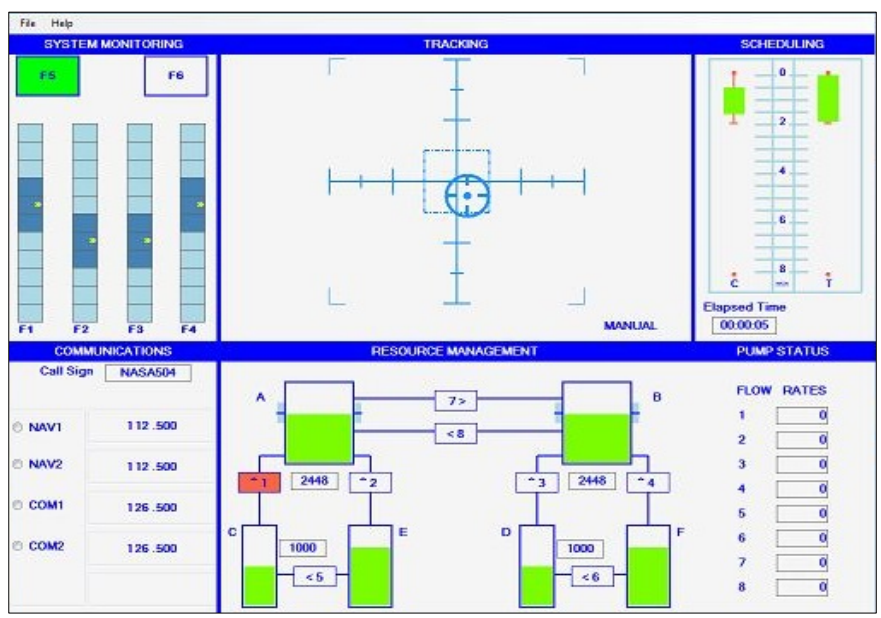

Figure 1. The MATB II interface, including clockwise from top left: monitoring, tracking, resource management and communications.

The MATB II platform (Figure 1) presents four distinct tasks to the operator. The monitoring task (upper left) requires operators to notice whether a red light has appeared. A compensatory tracking task (upper center) requires 
participants to use the joystick to continuously adjust the position of the reticle on a central point. In our instructions, we further noted this as keeping the dot within the circular reticle exactly on the cross of the $\mathrm{X}$ and $\mathrm{Y}$-axis.

The resource management task (lower center) is the third task, in which participants attempt to keep two central fuel tanks filled to threshold levels. The central tanks drain over time, but are refilled by activating directional pumps, connected to the remaining tanks. Pumps are under the control of the operator to be turned on or off unless they fail, in which case they are inoperably off until 30s has elapsed (when they remain off, but can now be turned on). Events in this task are pump failures, which require operators to compensate by using other pumps to maintain the levels.

A communications task (lower left) mimics an airtraffic controller task, wherein the participant listens for a call sign and a set of instructions. After instructions are given, the participant enters the information for two values communicated by the instructions (radio type and radio frequency) into the display.

\section{Procedure}

Participants were given ten minutes to read and then review a training manual presentation, before completing a two-minute MATB training trial that included all four active MATB task components. During training, and all following trials, participants were not allowed to make responses on the joystick or mouse other than with their dominant hand (i.e., no dual-handed performance, or hand switching, was ever allowed). Thus, when choosing to make responses with the joystick, participants had to have their dominant hand on the stick, and when switching to make responses in all other tasks, the same hand had to be moved to the mouse.

Before transitioning to testing trials, participants were randomly assigned to one of two tracking task difficulty conditions (easy or difficult). Difficulty of the tracking task was manipulated using tracking bandwidth and update rates. Participants were instructed to attempt to complete all tasks quickly and accurately, with equal priority for all tasks.

During each of the six test trials (each lasting 10 minutes), participants encountered alternating phases where tracking or communications tasks were active. These phases lasted one minute for tracking and 30 s for communications for a total of 6 phases of each type over each trial. Tracking and communication task periods never overlapped. During each of the six phases in each trial, participants were faced with an alternative task to potentially switch to, indicated by the occurrence of an outside task event (either a red light onset in the monitoring task, or one of two critical pump failures in the resource management task). Events occurred during early, middle, or late time epochs for each phase. Participants were given free choice to respond or ignore the events that occurred in equal amounts across epoch and by type over all six trials. Subjective paired task ratings (similar to Gutzwiller et al. 2014) were collected after the first MATB test trial, and then again after the final test trial. Following the completion of the final survey, participants also completed the attentional network task (ANT; Fan et al., 2002).
Tracking performance was measured by the mean squared error in pixel distance between the reticle and the centered position. Using this measurement, we were also able to construct a state space analysis of tracking performance for all tracking phases, wherein tracking error, and error rate, were examined (Wickens, 1986). State space analysis allowed us to assess the extent to which switches occurred at optimal switching points, within this otherwise continuous task. We defined an optimum switching point within the tracking task, as occurring when tracking was stabilized with low error and not diverging. In contrast, a non-optimal point was defined when tracking error was high and diverging. Switches away from tracking that occurred at optimal and non-optimal points were tallied, and thus, it was possible to determine the extent to which switches under these conditions were associated with executive control (as assessed by ANT).

The ANT test is designed to measure the activity of three attentional networks: alerting, orienting and conflict effects. Of interest was the conflict outcome, as was used previously in Arrington and Yates (2009), as it taps executive control processes. During the ANT task, participants are either cued (or not) to the potential location of a flanker task presentation. The ANT task uses arrows instead of letters; incongruent presentations show flanking arrows pointing in the opposite direction of the target arrow, and congruent presentations show arrows pointing the same way as the target (in the neutral condition, no surrounding arrows are presented). A central point was instructed to be fixated during intervening trial time. Participants were asked to judge whether the target (e.g., central) arrow was pointing left or right as quickly and accurately as possible.

Participants completed 24 practice trials (practice block) in the ANT task, receiving feedback only after the practice block. Participants then completed three test blocks of 96 trials with no feedback. Participants made their left and right responses using the naturally mapped left and right buttons of the mouse and their dominant hand. Although alerting and orienting values were also obtained from the cueing manipulations present in ANT, we focused on the executive control network assessed by the flanker task results.

\section{RESULTS}

\section{Individual Differences in Switching}

Pearson correlation coefficients were calculated between the conflict outcome executive control measure and average switch frequency for 49 participants, due to missing ANT data and one outlier. The flanker task component is the evaluation of the executive control network, and was measured by subtracting response times of the congruent trials from those of the incongruent trials. Thereby a measure is provided in which a larger difference implies increased distraction. Scores on this executive conflict measure $(M=$ $139, S D=42$ ) overall appear to be in the range of other values reported in Fan et al. (2002), and Arrington and Yates (2009). However, a Pearson correlation did not show a relationship between the executive control measure of ANT and switching overall in the MATB trials $(r=-.22, p=.13)$, though the 
direction does concur with Arrington and Yates (higher conflict RTs leading to reduced switching).

We further investigated whether the relationship differed by difficulty condition. Increasing task difficulty could bring out the hypothesized effect, given the increased demand for resources, and participants' variation in their ability to meet this demand when alternative task events occurred. The correlation was recalculated, split by tracking difficulty condition; however, no relationship was shown in either the easy condition $(r=-.06, p=.82)$ or the difficult condition $(r=-.29, p=.14)$. Given the moderate power of these examinations, a strong conclusion is not advised but the current data do not support a direct relationship. The size of the effect also suggests at most, the relationship between conflict resolution measures and general switching frequency is weak.

\section{State Space Analysis}

Overall, switching frequency may not be indicative of controlling attention. Executive control ability may be more closely related to switching when it is appropriate, or optimal, to do so - in particular by taking advantage of natural breakpoints in tasks. To answer this question, we examined the 27 participants in the difficult condition of the tracking task, during the first three trials, where natural breakpoints were more likely to be a factor in switching. A Pearson correlation showed that switches at optimal $(M=8.59, S D=$ $4.80)$, but not at non-optimal tracking task breakpoints $(M=$ $6.52, S D=2.93)$, were negatively correlated with executive measures from the Flanker task $(r=-.38, p=.05$; and $r=-.20$, $p=.33$, respectively). The significance in the former suggests that more control (shorter time to resolve response conflict) is related to increased switches when it is optimal to do so.

A split halves partition of participants' score on the conflict outcome portion of ANT produced two groups of low, and high reaction time (RT). Figure 2 presents the data subjected to a 2 (RT group) by 2 (optimality) ANOVA.

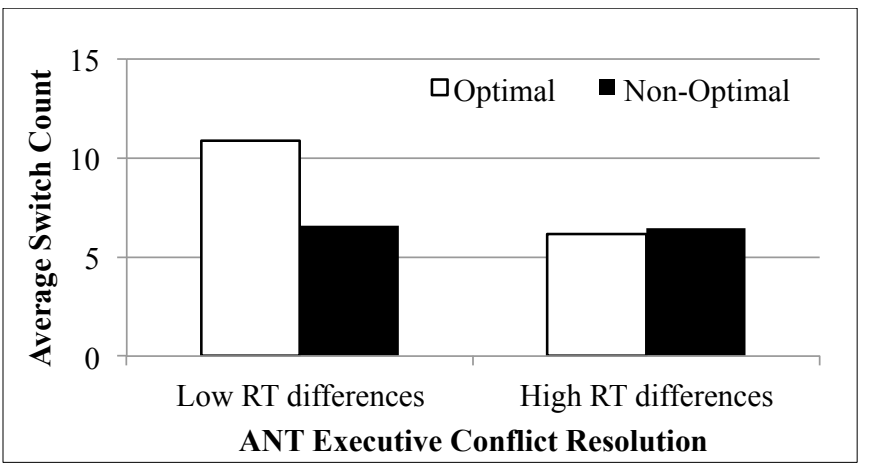

Figure 2. Executive control conditions and switches executed away from the ongoing tracking task during periods of optimality. Low RT difference $=$ higher executive network efficiency; High RT difference = lower efficiency.

While there were more switches in optimal conditions overall $\left(F(1,25)=5.47, p=.03, \eta_{\mathrm{p}}{ }^{2}=.18\right)$, switches interacted with group $\left(F(1,25)=7.30, p=.01, \eta_{\mathrm{p}}{ }^{2}=.23\right)$. The general main effect of more switching during optimal conditions was only observed for the high executive control group $(t(13)=3.12, p=.008)$, and not found in the low executive control group $(t(12)=-.32, p=.75)$. These results generally support our second hypothesis $(H 2)$, because improved conflict resolution ability appears to allow an individual to exploit more optimal breakpoints. The direction of the correlation is also partially consistent with that previously found by Arrington and Yates (2009): more executive control leads to more switches.

\section{DISCUSSION}

Switch frequency has been the primary unit of analysis in the task switching literature. In the current experiment, we included measures of switching at optimal, versus non-optimal times. The ability to resolve conflicts in response may be required when optimal times to switch arrive, and thus may be related to exploiting natural breakpoints. The results suggest overall that the measure of an executive network component of attention does not appear related to general task switching frequency, contrary to the basic findings of Arrington and Yates (2009). Thus, we do not replicate their findings with our complex, heterogeneous switching tasks without switching constraints on choice.

The tracking state space analysis was undertaken to examine how differences in ability may be related to "when" switches occur. We found that higher executive control (and thus, higher ability to resist interference) pointed to an increased likelihood to switch optimally - to exploit switch opportunity. These results address a gap that other studies have left open, and represent a new finding relevant for understanding multi-task management.

As far as the specific individual differences tapped in task switching (and especially choosing optimal switch points), we would also expect a relationship to exist with WM, based on how WM plays a role in Stroop interference resistance (e.g., Kane \& Engle, 2003), and the similarity, in this respect, to the Flanker task used here. Though Butler et al. (2011) found no relationship between WM and switching frequency, they did not examine the "opportunity" of a switch. A point for future research is to measure WM for comparison.

In the paradigm used in Arrington and Yates (2009), executive response conflict ability is tapped repeatedly during trials, because two tasks were repeatedly available for participants to choose between. In the current experiment, similar choices were present primarily as a function of alternative task events, which competed directly for attention. However, choices may also be present because of other tasks that require frequent attention, such as the management task, independent of specific task events; this suggests the relationship may extend beyond simple conflict resolution.

One reason our findings may be unique is that we have constrained task performance to sequential task switching, rather than "concurrency". Such constraints move performance from the multiple resources perspective (Wickens, 2002; 2008), to that of our sequential task switching choice model, STOM (Gutzwiller, Wickens, \& Clegg, 2014; Wickens et al., 2015). Though individual differences in executive functioning did exert some influence here, other 
individual differences may exert effects on task switching choice and switch "optimality". For example, individual differences in polychronicity (a preference to multitask) account for whether operators choose to multitask, in addition to impulsivity (e.g., König et al., 2010), a facet related to EC.

Individual differences in WM may be inversely related to switching behaviors (Sanbonmatsu et al., 2013), and WM also emerges as an important construct when keeping track of multiple goals (Altmann \& Trafton, 2002). However, as we alluded to above, these perspectives may miss the relationship between how opportune the switching was. After all, switches are not inherently "negative" behaviors, per se.

Differences in the frequency, and the ability to select opportune times to switch, may also arise from extensive practice. Experienced nurses, for example, perform better at resisting interruptions during key phases of an operation compared to novice nurses (Koh, Park, Wickens et al., 2011). Frequent heavy media multi-tasking may lead to reduced cognitive control on tasks with distracting components (Ophir et al. 2009). As operators do trend toward selecting optimal breakpoints in some task combinations (Janssen \& Brumby, 2010), and it could be (but is unlikely here) that aspects of resolving conflict can be learned.

In summary, components of executive attention do indeed relate to task switching behaviors, even in more realistic, multi-task management situations. These differences do not fully confirm the general correlational relationship found in basic research, but rather show how greater executive control relates to exploiting naturally optimal periods in ongoing task performance. In exploring time-ontask effects then, the variation in when "optimal" times to switch arise, and the operators' ability, may need to be taken into account.

\section{ACKNOWLEDGMENTS}

This work was supported by NASA under Grant NNX12AE69G (PI: Angelia Sebok), technical monitor Dr. Jessica Marquez and technical sponsor Dr. Brian Gore. RSG's contribution was also supported by a DoD SMART scholarship through the Navy's Space and Naval Warfare Systems Center Pacific. We thank Tyler Scott for aid in extracting data from MATB.

The views and opinions expressed here are those of the authors and do not reflect the official policy or position of any agency of the U.S. government.

\section{REFERENCES}

Altmann, E. M., \& Trafton, J. G. (2002). Memory for goals: an activationbased model. Cognitive Science, 26(1), 39-83.

Arrington, C. M., \& Yates, M. M. (2009). The role of attentional networks in voluntary task switching. Psychnomic Bulletin \& Review, 16(4), 660-5.

Banich, M. (2009). Executive function: the search for an integrated account. Current Directions in Psychological Science, 18(2), 89-94.

Bogunovich, P., \& Salvucci, D. D. (2008). Inferring multitasking breakpoints from single-task data. Proc of the Cognitive Science Society, 1732-37.

Butler, K. M., Arrington, C. M., \& Weywadt, C. (2011). Working memory capacity modulates task performance but has little influence on task choice. Memory \& Cognition, 39(4), 708-724.

Chou, C., Madhavan, D., \& Funk, K. (1996). Studies of cockpit task management errors. The Intnl Jrnl of Avtn Psychology, 6(4), 307-320.
Comstock, J. R., \& Arnegard, R. J. (1992). The Multi-Attribute Task Battery for human operator workload and strategic behavior research. NASA Technical Memorandum 104174.

Dehais, F., Causse, M., \& Tremblay, S. (2011). Mitigation of conflicts with automation: Use of cognitive countermeasures. Human Factors, 53(5), $448-460$.

Demanet, J., Verbruggen, F., Liefooghe, B., \& Vandierendonck, A. (2010). Voluntary task switching under load: contribution of top-down and bottom-up factors in goal-directed behavior. Psychonomic Bulletin \& Review, 17(3), 387-393.

Dismukes, R., \& Berman, B. (2010). Checklists and monitoring in the cockpit: Why crucial defenses sometimes fail. NASA/TM-2010-216396

Eriksen, B., \& Eriksen, C. (1974). Effects of noise letters upon the identification of a target letter in a nonsearch task. Perception \& Psychophysics, 16, 143-149.

Fan, J., McCandliss, B. D., Sommer, T., Raz, A., \& Posner, M. I. (2002). Testing the efficiency and independence of attentional networks. Journal of Cognitive Neuroscience, 14(3), 340-347.

Funk, K. (1991). Cockpit task management: preliminary definitions, nonnative theory, error taxonomy, and design recommendations. International Journal of Aviation Psychology, 1(4), 271-285.

Gutzwiller, R. S. (2014). Switch choice in applied multi-task management. Doctoral Dissertation, 1-179.

Gutzwiller, R. S., Wickens, C. D., \& Clegg, B. A. (2014). Workload overload modeling: An experiment with MATB II to inform a computational model of task management. Procedings of the Human Factors and Ergonomics Society Annual Meeting, 58(1), 849-853.

Janssen, C. P., \& Brumby, D. P. (2010). Strategic adaptation to performance objectives in a dual-task setting. Cognitive Science, 34(8), 1548-1560.

Kane, M. J., \& Engle, R. W. (2003). Working-memory capacity and the control of attention: the contributions of goal neglect, response competition, and task set to Stroop interference. Journal of Experimental Psychology: General, 132(1), 47-70.

Koh, R. Y. I., Park, T., Wickens, C. D., Ong, L. T., \& Chia, S. N. (2011). Differences in attentional strategies by novice and experienced operating theatre scrub nurses. Journal of Experimental Psychology: Applied, 17(3), 233-246.

König, C. J., Oberacher, L., \& Kleinmann, M. (2010). Personal and situational determinanets of multitasking at work. Journal of Personnel Psychology, 9(2), 99-103.

Kurzban, R., Duckworth, A., Kable, J. W., \& Myers, J. (2013). An opportunity cost model of subjective effort and task performance. Behavioral and Brain Sciences, 36(6), 661-679.

Loukopoulos, L., Dismukes, R., \& Barshi, I. (2009). The multitasking myth. Farnham: Ashgate Publishing, Ltd.

McLeod, C. (1991). Half a century of research on the Stroop effect: An integrative review. Psychological Bulletin, 109(2), 163-203.

Ophir, E., Nass, C., \& Wagner, A. D. (2009). Cognitive control in media multitaskers. PNAS, 106(37), 15583-7.

Sanbonmatsu, D. M., Strayer, D. L., Medeiros-Ward, N., \& Watson, J. M. (2013). Who multi-tasks and why? Multi-tasking ability, perceived multi-tasking ability, impulsivity, and sensation seeking. PloS One, $8(1)$, e54402.

Santiago-Espada, Y., Myer, R. R., Latorella, K. A., \& Comstock, J. R. (2011). The Multi-Attribute Task Battery II (MATB-II): A User's Guide.

Weywadt, C. R. B., \& Butler, K. M. (2013). The role of verbal short-term memory in task selection: how articulatory suppression influences task choice in voluntary task switching. Psych Bul \& Rvw, 20(2), 334-340.

Wickens, C. D. (1986). The effects of control dynamics on performance. In B. KR, L. Kaufman, \& J. Thomas (Eds.), Handbook of Perception and Performance (pp. 39-60). New York: Wiley.

Wickens, C. D. (2002). Multiple resources and performance prediction. Theoretical Issues in Ergonomics Science, 3(2), 159-177.

Wickens, C. D. (2008). Multiple resources and mental workload. Human Factors, 50(3), 449-455.

Wickens, C. D., \& Alexander, A. L. (2009). Attentional Tunneling and Task Management in Synthetic Vision Displays. The International Journal of Aviation Psychology, 19(2), 182-199.

Wickens, C. D., Gutzwiller, R. S., \& Santamaria, A. (2015). Discrete task switching in overload: A meta-analysis and a model. International Journal of Human-Computer Systems, 79, 79-84.

Wickens, C. D., Hollands, J., Banbury, S., \& Parasuraman, R. (2013). Engineering psychology and human performance (4th ed.). Upper Saddle River, NJ: Pearson. 